\title{
Tandem MS for environmental and food analysis
}

\author{
Damià Barceló • Mira Petrovic
}

Published online: 27 August 2010

(C) Springer-Verlag 2010

This special issue contains selected papers presented at the sixth annual LC/MS/MS Workshop on Environmental Applications and Food Safety. Focus on Emerging Substances held on 25-26 February 2010 in Barcelona, Spain.

Following the success of the previous five annual workshops on liquid chromatography-tandem mass spectrometry (LC-MS/MS) for screening and trace-level quantitation in environmental and food samples (Barcelona, June 2005; Barcelona, September 2006; Guelph, April 2007; Barcelona, February 2008; Toronto, April 2009), this sixth workshop was jointly organized by CSIC - Consejo Superior de Investigaciones Cientificas (Barcelona, Spain), Waters Corporation (Manchester, UK), Environment Canada and the Institut d'Estudis Catalans (Barcelona, Spain) in collaboration with the Spanish Society for Mass Spectrometry (SEEM) and the Catalan Institute for Water Studies (ICRA).

In the course of the workshop, 25 keynote lectures and 37 poster presentations were presented. The principal topics were practical aspects of the usefulness of tandem massspectrometric techniques for screening and quantitation of organic contaminants in the environmental and food samples. This year, the focus was on the analysis of emerging substances, such as nanoparticles, siloxanes, perfluorinated compounds, pharmaceuticals, drugs of abuse and polar pesticides.

Over the last 20 years, liquid chromatography-mass spectrometry techniques have advanced dramatically in their sensitivity, specificity and reliability. Detection of sub-parts per trillion concentrations is becoming routine for many organic

D. Barceló $(\square) \cdot$ M. Petrovic

Department of Environmental Chemistry, IDAEA-CSIC,

c/Jordi Girona 18-26,

08034 Barcelona, Spain

e-mail: dbcqam@cid.csic.es

M. Petrovic

e-mail: mpeqam@cid.csic.es analytes and methods achieving detection of a few hundred femtograms of some analytes have been reported. Such progress is mostly due to development of LC-MS/MS techniques, which are today the methods of choice for the determination of trace organic analytes in environmental and food samples. Swift growth in the use of LC-MS/MS for the analysis of organic contaminants in environmental and food matrices has been compelled by the need for high-quality data on their occurrence in such complex matrices at very low concentrations.

At the workshop and in this special issue, some of the most recent trends in the LC-MS/MS analysis of emerging contaminants in environmental and food matrices were covered, such as:

- Advanced sample preparation technologies for food and environmental analysis such as the use of molecularly imprinted polymers and other selective solid-phaseextraction cartridges, including online precolumn technology coupled to LC-MS/MS systems

- The discovery of new metabolites and transformation products of biotic and abiotic processes by using a combination of the various advanced mass-spectrometric techniques

- Development of generic analytical protocols that permit simultaneous determination of multiclass compounds, including both parent compounds and their metabolites

The papers in this special issue also give an overview of the advantages, comparative usefulness and complementarities of cutting-edge technologies, tandem and hybrid mass spectrometry instruments, such as quadrupole-linear ion trap, quadrupole-time-of-flight and Orbitrap, in the quantitative and qualitative determination of complex environmental and food samples. Large numbers of applications for environmental samples, basically water and soil/sediment, and food samples, such as fruits, vegetables, juices and meat, 
including a broad range of contaminants and their degradation compounds such as polar pesticides, pharmaceuticals, drugs of abuse, perfluorinated compounds, microcystins and brominated compounds, are presented.

Finally, we would like to thank all the authors of this selection of articles for their participation at the workshop and for their written contributions that made this special issue possible.

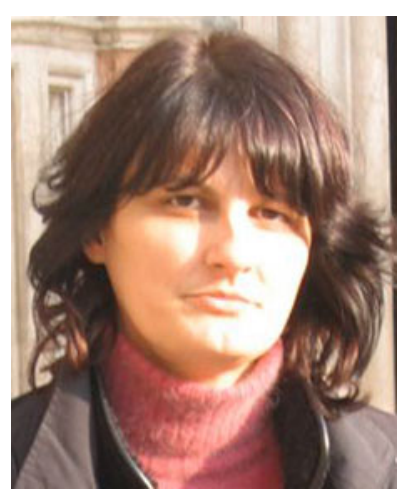

Mira Petrovic is an ICREA research professor at the Department of Environmental Chemistry, IDAE-CSIC, Barcelona, Spain. Her main expertise is in the field of analytical environmental chemistry, specifically analysis of organic micropollutants by advanced mass-spectrometric techniques and the study of their fate and behaviour in the aquatic environment and during wastewater and drinking water treatment.

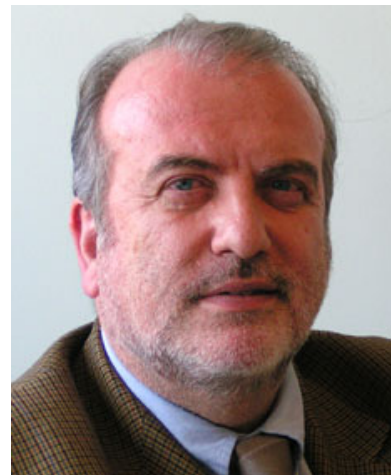

Damiá Barceló is a full research professor at the Environmental Chemistry Department at IDAEA-CSIC in Barcelona, Spain, Director of the Catalan Institute for Water Studies (ICRA) in Girona, Spain, and Visiting Professor at the King Saud University, Saudi Arabia. $\mathrm{He}$ has been President of the Spanish Society of Mass Spectrometry (SEEM) since 2006. His research focuses on method development and the monitoring and fate of priority, new and emerging pollutants in water, using gas chromatography and liquid chromatography techniques coupled with advanced tandem and hybrid mass-spectrometric analysis combined with effect studies using bioassays and biosensors. $\mathrm{He}$ is the coordinator the project INNOVA-MED Innovative Processes and Practices for Wastewater Treatment and Reuse in the Mediterranean Region funded by the European Union and the project Assessing and Predicting Effects on Water Quantity and Quality in Iberian Rivers (SCARCE) funded by the CONSOLIDER-INGENIO 2010 programme of the Spanish Ministry of Science and innovation 